\title{
STRUCTURAL ASPECTS AND FLORISTIC SIMILARITY AMONG TROPICAL DRY FOREST FRAGMENTS WITH DIFFERENT MANAGEMENT HISTORIES IN NORTHERN MINAS GERAIS, BRAZIL ${ }^{1}$
}

\author{
Daniel Meira Arruda², Diego Oliveira Brandão ${ }^{3}$, Fernanda Vieira Costa 4 , Gláucia Soares Tolentino², \\ Reinaldo Duque Brasil ${ }^{2}$, Santos D’ Ângelo Neto ${ }^{5}$ e Yule Roberta Ferreira Nunes ${ }^{5}$
}

\begin{abstract}
In order to produce useful knowledge to the initiatives of protection and management of forest fragments, more specifically for tropical dry forests which suffer with frequent anthropic activities, and due to the lack of specific studies, this article aimed describe the structure and the floristic similarity among three areas of dry forest with different management histories. The study was developed in Capitão Enéas municipality, Northern Minas Gerais, Brazil, where three fragments were evaluated, being one in regeneration for 30 years, another submitted to occasional fire and the third with selective cut in small scale. The sampling was developed through the point quarter method considering all the alive phanerophyte individuals with circumference at breast height $(\mathrm{CBH}) \geq 15 \mathrm{~cm}$. In the three fragments, 512 individuals, distributed in 60 species, 47 genera, and 23 families were sampled. The most representative families were Fabaceae (26), Anacardiaceae (4), Bignoniaceae (3) and Combretaceae (3). However, fourteen families were represented by only one species. Only eight species were common to all fragments - Myracrodruon urundeuva standed out with $26.9 \%$ of all sampled individuals - while a great number of species were exclusive of each fragment. The floristic and structural differences between the fragments are possibly related to the history and intensity of management in each area besides the topography variations and the presence or absence of limestone outcrops. These results show the importance of each fragment, indicating that the loss of anyone would cause negative impacts on the regional flora and consequently to the associated biodiversity.
\end{abstract}

Key-words: Seasonally Tropical Dry Forest, Phytossociology and Point Quarter Method.

\section{ASPECTOS ESTRUTURAIS E SIMILARIDADE FLORÍSTICA ENTRE FRAGMENTOS DE FLORESTA TROPICAL SECA COM DIFERENTES HISTÓRICOS DE MANEJO NO NORTE DE MINAS GERAIS, BRASIL}

RESUMO - A fim de gerar conhecimento útil às iniciativas de proteção e manejo de fragmentos florestais, especificamente para florestas tropicais secas, que sofrem com frequentes atividades antrópicas e com a falta de estudos específicos, este artigo objetivou descrever a estrutura e a similaridade florística de três áreas de floresta estacional decidual em diferentes históricos de manejo. O estudo foi desenvolvido em Santana da Serra, distrito de Capitão Enéas, Norte de Minas Gerais, Brasil, onde foram avaliados três fragmentos, sendo um em regeneração há 30 anos, outro submetido a fogo ocasional e um terceiro com corte seletivo em pequena escala. A amostragem foi desenvolvida através do método de pontos quadrantes, considerando todos os indivíduos fanerófitos vivos com circunferência à altura do peito $(C A P) \geq 15 \mathrm{~cm}$. Nos três fragmentos foram amostrados 512 indivíduos distribuídos em 60 espécies, 47 gêneros e 23 famílias. As famílias mais representativas foram Fabaceae (26), Anacardiaceae (4), Bignoniaceae (3) e Combretaceae (3). Entretanto, 14 famílias foram representadas

\footnotetext{
${ }^{1}$ Recebido em 22.04.2009 e aceito para publicação em 25.08.2010.

${ }^{2}$ Programa de Pós-Graduação em Botânica da Universidade Federal de Viçosa, Viçosa, MG. E-mail: <arruda.dm@hotmail.com>, <glaucia.tolentino@gmail.com>e <rduquebrasil@yahoo.com.br>

${ }^{3}$ Programa de Pós-Graduação em Ecologia do Instituto Nacional de Pesquisa da Amazônia (INPA), Manaus-AM. E-mail: <dbrandao13@gmail.com>

${ }^{4}$ Bióloga, mestre em Biologia da Conservação pela Universidade Estadual de Montes Claros. E-mail: <fecostabio@gmail.com>. ${ }^{5}$ Departamento de Biologia Geral da Universidade Estadual de Montes Claros, Montes Claros-MG. E-mail: <santosdangelo@bol.com.br> e <yule.nunes@unimontes.br>.
} 


\begin{abstract}
por apenas uma espécie. Apenas oito espécies foram comuns aos fragmentos, destacando-se Myracrodruon urundeuva que representou $26,9 \%$ de todos os indivíduos amostrados, enquanto que um grande número de espécies foi exclusivo a cada fragmento. As grandes diferenças florísticas e estruturais existentes entre os fragmentos estão possivelmente relacionadas ao histórico e intensidade de manejo em cada área, juntamente com as variações do terreno e a presença ou ausência de afloramentos de calcários. Esses resultados denotam a importância de cada fragmento, uma vez que a perda de um deles acarretaria impactos negativos sobre a flora regional e, consequentemente, à biodiversidade associada.
\end{abstract}

Palavras-chave: Floresta Estacional Decidual, Fitossociologia e Ponto-quadrante.

\section{INTRODUCTION}

Tropical Dry Forests (TDF) are formations subject to a well defined climatic seasonality alternating rainy and dry periods. In the dry season more than $50 \%$ of TDF individuals show themselves completely without leaves (VELOSO, 1991). Usually, dry forests presents lower values of height and basal area when compared to tropical humid forests. The productivity occurs mainly during the rainy season when the litterfall accumulated during the dry season decomposes and increases the amount of organic matter to the soil (MURPHY and LUGO, 1986; SCARIOT and SEVILHA, 2005; PENNINGTON et al., 2005). The biological activity synchronized to the water availability makes the rain seasonality an essential ecological factor for the functioning of these ecosystems (MURPHY and LUGO, 1986).

The distribution of TDFs is spread as continuous outline or as natural patches surrounded by another kind of vegetation (SCARIOT and SEVILHA, 2005). According to these authors, in Brazil these formations occur mainly under the domain of Cerrado, Caatinga, Atlantic and Amazonian biomes. Due to its wide occurrence, these forests undergo different seasonality regimens in precipitation volume and temperature besides being submitted to different topographies and physical and chemical soil characteristics (SCARIOT and SEVILHA, 2005). The gradient of environmental factors associated to the floristic aspects of the adjacent vegetations makes the species composition of this formation peculiar to each area where it occurs (PEDRALLI, 1997).

According to Kuhlmann (1951), the dry forests on limestone outcrops in Northern Minas Gerais represent an extension of the Caatinga floristic domain because they have similar xerophytes species. The Northern area of Minas Gerais constitutes part of the so-called “Polígono das Secas” (Drought Polygon) (BRANDÃO and GAVILANES, 1994), a region of great biological importance due to the presence of transition zones between the Cerrado, Caatinga and Atlantic Forest domains (BRANDÃO and GAVILANES, 1994).

In this region, the advance of the agribusiness technologies and management practices has converted the TDFs into pastures and agricultural landscapes. Moreover, the landscape change caused by mining companies is frequent in areas of dry forest on limestone outcrops (BRANDÃO et al., 2007). These and others human activities have promoted a $4.34 \%$ reduction of the tropical forests of the São Francisco basin, only in the period from 2003 to 2005 (SCOLFORO and CARVALHO, 2006). Consequently, these forests are normally constituted by a number of isolated fragments with diverse management histories and representing different sucessional stages (ARROYO-MORA et al., 2005). In this context, the environmental complexity varies from initial stages, with simpler structure and lower diversity, to advanced stages, with more complex structure and higher diversity (KALÁCSKA et al., 2004; MADEIRA et al., 2009).

Due to the crescent impacts caused by the intense land use management, mostly in last two decades (WERNECK et al., 2000), and to the scarcity of scientific knowledge on these ecosystems (SÁNCHEZ-AZOFEIFA et al., 2005), the structural and floristic surveys are an important tool for understanding the consequences of the disturbances derived from the wood extraction and fragmentation (SILVA and SCARIOT, 2004). In this way, in order to generate useful knowledge for the initiatives of protection and sustainable management of TDF fragments, this work aimed to describe the structure and the floristic similarity of three dry forest areas with different management histories.

\section{MATERIALAND METHODS}

This work was carried out in Santana da Serra, a rural district of Capitão Enéas municipality, located in Northern Minas Gerais. This region is predominated 
by TDF formations (also called "Matas Secas") on limestone outcrop and/or slate or without direct association with these rocks. The local climate is marked by a long dry season that lasts five months in which the monthly precipitation is inferior to $60 \mathrm{~mm}$. The pluviometric average is $1000 \mathrm{~mm} /$ year and the average temperature is $23^{\circ} \mathrm{C}$ (ANTUNES, 1994). The soils of the region are mostly Latosol and Ultisol types, both red-yellow, red-dark Ultisol and Cambisol, all eutrophic with clayey texture (NAIME, 1994).

Three fragments, about $3 \mathrm{~km}$ far from each other, were chosen for the sampling. Locally, these areas are known as “Morro da Cotia” (1608'59”'S 4341'59”'W),

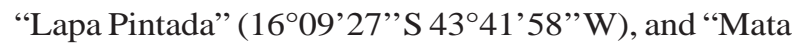
do Neco” (1609'05'” $43^{\circ} 43^{\prime} 19^{\prime}$ ' W). According to information of the local inhabitants, "Morro da Cotia” (MC) has been in regeneration process for about 30 years. This area has approximately 3.3 ha and before being abandoned, MC has suffered cut followed by conversion into pastures. "Lapa Pintada” (LP) has approximate area of 2.9 ha and is protected by the local inhabitants due to the presence of rupestrian paintings in limestone caves. Although this, reports of local inhabitants and fire vestiges indicates small disturbances in the area. The terrain of LP is very irregular with a great amount of limestone exposure. "Mata do Neco" (MN) has approximate area of 11.9 ha and according to the owner did not suffer disturbances in the last 50 years except selective cut in small scale. This fragment has no evidence of limestone outcrops.

In order to evaluate the structure of the tree community, the point quarter method was adopted (COTTAM and CURTIS, 1956), due to the versatility of its execution in irregular areas with the presence of rocky outcrops. Eight points 20 m equidistant among themselves were distributed along $180 \mathrm{~m}$ linear transects which were $20 \mathrm{~m}$ distanced of each other. Six transects were launched in MC, three in LP, and seven in MN, totalizing 48, 24 and 56 points respectively. The amount of transects varied among the areas due to access difficulty to the vegetation in certain places and due to the size of the fragments. In each quadrant the alive phanerophyte individuals with circumference at breast height - measured $1.30 \mathrm{~m}$ above ground - $(\mathrm{CBH}) \mathrm{e}$ " $15 \mathrm{~cm}$ were included. For each individual, the CBH was taken and the values of total height were also surveyed.
The botanical material collected was deposited in the Herbário Montes Claros (HMC) of the Universidade Estadual de Montes Claros (UNIMONTES). The recognition of the species was made through comparisons with their botanical collection and specialized literature using the APG II system (APG II, 2003). The phytossociological parameters, Shannon-Wiener diversity index ( $\left.\mathrm{H}^{\prime}\right)$ and evenness of Pielou (J') were calculated through FITOPAC 1.0 software (SHEPHERD, 1995). The similarity matrix was obtained using Jaccard similarity index (MUELLERDOMBOIS and ELLENBERG, 1974).

\section{RESULTS}

The number of 512 individuals, distributed in 60 species, 47 genera and 23 families were sampled, besides five unidentified individuals. The most representative families were Fabaceae (26 species), Anacardiaceae (4), Bignoniaceae (3) and Combretaceae (3). These four families corresponded $55 \%$ of the sampled species. Fourteen families were represented by only one species and the other ones by two. The most representative specie in the three areas was Myracrodruon urundeuva, corresponding to $26.9 \%$ of the total sampled individuals. Combretum duarteanum, C. leprosum, Machaerium acutifolium, Acacia sp., Schinopsis brasiliensis, Sapium obovatum and Bougainvillea praecox were also common to the three sampled areas (Table 1).

In "Morro da Cotia” (MC), 36 species were registered plus two unidentified individuals (Tables 1 and 2). The five species with greatest importance value (IV) in this area were M. urundeuva, (represented by $52.6 \%$ of the individuals), C. leprosum (6.2\%), Tabebuia roseoalba (5.7\%), S. brasiliensis (2.1\%) and Piptadenia gonoacantha (4.2\%). Among the species sampled in this fragment, 20 (52.6\%) were exclusive of MC and amongst them, 17 were represented by only one individual. The vertical structure of this area has average height of $7.2 \mathrm{~m}$ and $M$. urundeuva $(8.3 \mathrm{~m})$ is the main component of the canopy, followed by Anadenanthera colubrina (7.3 m), C. leprosum (5.8 m) and T. roseoalba (5.2 m) (Figure 1). The total density of the area is 909.4 ind.ha ${ }^{-1}$, with Shannon-Wiener diversity index ( $\mathrm{H}^{\prime}$ ) equal to 2.21 and Pielou's index of evenness ( $\mathrm{J}$ ') equal to 0.61 .

Revista Árvore, Viçosa-MG, v.35, n.1, p.131-142, 2011 
Table 1 - List of species sampled in Santana da Serra, Capitão Enéas, MG, with its respective ecological groups (LORENZI, 1992; 1998; CARVALHO, 2003; 2006): pioneering (PI), secondary initial (SI), climax light demanding (CL); occurrence (OLIVEIRA-FILHO, 2006): Cerrado lato sensu (CE), Caatinga (CA), Dry Forest (DF), gallery forest (FG), Atlantic Forest (AF); and occurrence in the sampled fragments in the present study: Morro da Cotia (MC), Lapa Pintada (LP) and Mata do Neco (MN).

Tabela 1 - Lista de espécies amostradas em Santana da Serra, Capitão Enéas, MG, com seus respectivos grupos ecológicos (LORENZI, 1992; 1998; CARVALHO, 2003; 2006): pioneira (PI), secundária inicial (SI), clímax exigente de luz (CL); ocorrência (OLIVEIRA-FILHO, 2006): Cerrado (lato sensu) (CE), Caatinga (CA), Floresta Seca (DF), Floresta de galeria (FG), Mata Atlântica (AF); e registro nos fragmentos amostradas no presente trabalho: Morro da Cotia (MC), Lapa Pintada (LP) e Mata do Neco (MN).

Family/Species

ANACARDIACEAE

Myracrodruon urundeuva Allemão

Schinopsis brasiliensis Engl.

Spondias macrocarpa Engl.

Spondias tuberosa Arruda

ANNONACEAE

Annona sp.

APOCYNACEAE

Aspidosperma pyrifolium Mart.

Tabernaemontana solanifolia A.DC.

ARALIACEAE

Aralia warmingiana (Marchal) J.Wen

ARECACEAE

Syagrus oleracea (Mart.) Becc.

BIGNONIACEAE

Tabebuia ochracea (Cham.) Standl.

Tabebuia roseoalba (Ridl.) Sandwith.

Tabebuia sp.

BURSERACEAE

Commiphora leptophloeos (Mart.) J.B.Gillett

CACTACEAE

Cereus jamacaru DC.

CANNABACEAE

Celtis iguanaea (Jacq.) Sarg.

COMBRETACEAE

Combretum duarteanum Cambess.

Combretum leprosum Mart.

Terminalia sp.

EUPHORBIACEAE

Cnidoscolus pubescens Pohl

Sapium obovatum Klotzsch ex MüllArg.

FABACEAE

Acacia polyphylla DC.

Acacia sp.

Acosmium fallax (Taub.) Yakovlev

Albizia niopoides (Spruce ex Benth.) Burkart

Anadenanthera colubrina (Vell.) Brenan

Bauhinia cheilantha (Bong.) Steud.

Bauhinia forficata Link

Bauhinia rufa (Bong.) Steud.

Chloroleucon tortum (Mart.) Pitter

Deguelia costata (Benth.) Az.-Tozzi

Ecological group

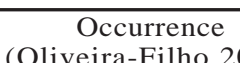

(Oliveira-Filho 2006)

SI, CL

SI

SI

P I

P I

P I

P I

P I

PI, CL

PI

PI, SI

PI, SI

\section{DF, CE, AF, CA, FG \\ DF, CE, FG AF}

DF, CE, CA

DF, CE, AF, CA

AF, CE, FG

AF, CE, DF, FG

AF, CE, DF, FG

AF, CE, DF, FG

AF, CE, DF, FG

AF, CE, DF, CA

AF, CE, DF, CA

AF, CE, FG

CE, DF

CE, DF, FG

DF, CA

$\mathrm{AF}$

AF, CE, DF, FG

DF, CA

AF, CE, DF, FG

AF, CE, DF, FG, CA

DF, CA

AF, CE, DF, FG

AF, CE, FG

$A F, C E, D F$

P I

AF, CE, DF, FG

Ocurrence in this study

MC, LA, MN
MC, LP, MN
MN
MC
MN
MC, LP
MN
MN
MN
MN
MC, LA
MC
MC, LA
MC
MC
MC, LA, MN
MC, LA, MN
LA
MN

MC, LP, MN

L P

MC, LP, MN

$\mathrm{MN}$

MN

MC, LP

$\mathrm{MC}$

LP, MN

LP

MC

L P 
Tabela 1 - Cont.

Table 1 - Cont.

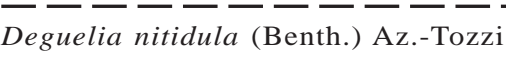

Erythrina velutina Willd.

\author{
P I
}

SI

Goniorrhachis marginata Taub.

Leucochloron incuriale (Vell.) Barneby \& J.W.

Grimes

Lonchocarpus campestris Mart. Ex Benth.

Lonchocarpus montanus Az.-Tozzi

Machaerium acutifolium Vogel

Machaerium scleroxylon Tul.

Piptadenia gonoacantha (Mart.) J.F.Macbr.

Platymiscium blanchetii Benth.

Platymiscium floribundum Vogel

Platypodium elegans Vogel

Pterogyne nitens Tul.

Senna macranthera (Collad.) H.S.Irwin

\& Barneby

Sweetia fruticosa Spreng.

Fabaceae sp.

MALVACEAE

Cavanillesia arborea (Willd.) K.Schum.

Guazuma ulmifolia Lam.

MELIACEAE

Trichilia claussenii C.DC.

MORACEAE

Maclura tinctoria (L.) Steud.

MYRTACEAE

Campomanesia sp.

NYCTAGINACEAE

Bougainvillea praecox Griseb.

POLYGONACEAE

Coccoloba schwackeana Lindau

RHAMNACEAE

Ziziphus joazeiro Mart.

RUBIACEAE

Chomelia sericea Müll.Arg.

Randia armata (Sw.) DC.

SALICACEAE

Casearia decandra Jacq.

SAPINDACEAE

Allophylus edulis (L.C.Rich.) A.Rich. ex DC.

Talisia esculenta (A.St.-Hil.) Radlk.

VOCHYSIACEAE

Callisthene major Mart.

NÃO INDENTIFICADAS

sp. 1

sp. 2

sp. 3

sp. 4

sp. 5

PI, SI, CL

P I

PI, SI

PI, SI, CL 
area presented four exclusive species and among them only Deguelia costata has more than one individual. The canopy of LP area is mostly constituted by $A$. colubrina $(9.7 \mathrm{~m})$ and the understory by C. leprosum (6.7 m), P. gonoacantha $(6.3 \mathrm{~m})$ and T. roseoalba $(6.0 \mathrm{~m})$ (Figure 1). The average of the vertical structure is 7.9 $\mathrm{m}$ and the total density is 1496 ind.ha ${ }^{-1}$.
In “Mata do Neco” (MN) 32 species were sampled, besides three unidentified individuals (Tables 1 and 4), of which 20 (57.1\%) were exclusive of this area. Among these exclusive species, nine were sampled only once. The five species with greatest IV were Leucochloron incuriale (23.6\% of the sampled individuals), Goniorrhachis marginata (12.9\%), $M$.

Table 2 - List of species sampled in Morro da Cotia dry forest fragment, Capitão Enéas, MG, and its phytosociological parameters, in decreasing sequence of importance value (IV). $\mathrm{N}=$ number of sampled individuals; $\mathrm{BA}=$ basal area; $\mathrm{H}_{\max }=$ maximum height; $\mathrm{AD}=$ absolute density; $\mathrm{RD}=$ relative density; $\mathrm{ADo}=$ absolute dominance; $\mathrm{RDo}=$ relative dominance; $\mathrm{AFr}=$ absolute frequency; $\mathrm{RFr}=$ relative frequency.

Tabela 2 - Lista de espécies amostradas no Morro da Cotia, Capitão Enéas, MG, e seus parâmetros fitossociológicos, em ordem decrescente de valor de importância (IV). $N=$ número de indivíduos amostrados; $B A=$ área basal; $H_{\max }=$ altura máxima; $A D=$ densidade absoluta; $R D=$ densidade relativa; $A D o=$ dominância absoluta; $R D o=$ dominância relativa; $A F r=$ frequência absoluta; $R F r=$ frequência relativa .

\begin{tabular}{|c|c|c|c|c|c|c|c|c|c|c|}
\hline Species & $\mathrm{N}$ & $\mathrm{BA}\left(\mathrm{m}^{2}\right)$ & $\mathrm{H}_{\max }(\mathrm{m})$ & $\mathrm{AD}$ & $\mathrm{RD}$ & ADo & RDo & AFr & RFr & IV \\
\hline Myracrodruon urundeuva & 101 & 2,711 & 16,0 & 478,4 & 52,6 & 12,84 & 75,80 & 89,60 & 35,80 & 164,00 \\
\hline Combretum leprosum & 12 & 0,072 & 10,0 & 56,8 & 6,3 & 0,34 & 2,02 & 18,80 & 7,50 & 15,80 \\
\hline Tabebuia roseoalba & 11 & 0,030 & 8,0 & 52,1 & 5,7 & 0,14 & 0,83 & 12,50 & 5,00 & 11,60 \\
\hline Schinopsis brasiliensis & 4 & 0,204 & 9,0 & 18,9 & 2,1 & 0,97 & 5,71 & 8,33 & 3,33 & 11,10 \\
\hline Piptadenia gonoacantha & 8 & 0,031 & 10,0 & 37,9 & 4,2 & 0,15 & 0,86 & 12,50 & 5,00 & 10,00 \\
\hline Randia armata & 5 & 0,015 & 9,0 & 23,7 & 2,6 & 0,07 & 0,42 & 10,40 & 4,17 & 7,19 \\
\hline Machaerium acutifolium & 4 & 0,037 & 8,0 & 18,9 & 2,1 & 0,17 & 1,02 & 8,33 & 3,33 & 6,44 \\
\hline Acacia sp. & 5 & 0,036 & 8,0 & 23,7 & 2,6 & 0,17 & 1,00 & 6,25 & 2,50 & 6,10 \\
\hline Anadenanthera colubrina & 3 & 0,049 & 8,0 & 14,2 & 1,6 & 0,23 & 1,38 & 6,25 & 2,50 & 5,44 \\
\hline Zizyphus joazeiro & 1 & 0,120 & 5,0 & 4,7 & 0,5 & 0,57 & 3,35 & 2,08 & 0,83 & 4,70 \\
\hline Bougainvillea praecox & 2 & 0,053 & 6,0 & 9,5 & 1,0 & 0,25 & 1,48 & 4,17 & 1,67 & 4,19 \\
\hline Commiphora leptophloeos & 3 & 0,022 & 6,5 & 14,2 & 1,6 & 0,10 & 0,61 & 4,17 & 1,67 & 3,84 \\
\hline Callisthene major & 3 & 0,015 & 6,0 & 14,2 & 1,6 & 0,73 & 0,43 & 4,17 & 1,67 & 3,66 \\
\hline Guazuma ulmifolia & 2 & 0,017 & 9,0 & 9,5 & 1,0 & 0,08 & 0,48 & 4,17 & 1,67 & 3,19 \\
\hline Celtis iguanaea & 2 & 0,012 & 6,0 & 9,5 & 1,0 & 0,06 & 0,33 & 4,17 & 1,67 & 3,04 \\
\hline Chomelia sericea & 2 & 0,011 & 5,5 & 9,5 & 1,0 & 0,05 & 0,30 & 4,17 & 1,67 & 3,01 \\
\hline Aspidosperma pyrifolium & 2 & 0,007 & 6,0 & 9,5 & 1,0 & 0,03 & 0,19 & 4,17 & 1,67 & 2,90 \\
\hline Sapium obovatum & 2 & 0,007 & 7,3 & 9,5 & 1,0 & 0,03 & 0,20 & 4,17 & 1,67 & 2,90 \\
\hline Platymiscium floribundum & 1 & 0,024 & 9,0 & 4,7 & 0,5 & 0,11 & 0,66 & 2,08 & 0,83 & 2,01 \\
\hline Combretum duarteanum & 1 & 0,018 & 7,0 & 4,7 & 0,5 & 0,08 & 0,49 & 2,08 & 0,83 & 1,84 \\
\hline Lonchocarpus montanus & 1 & 0,014 & 8,0 & 4,7 & 0,5 & 0,07 & 0,40 & 2,08 & 0,83 & 1,76 \\
\hline Spondias tuberosa & 1 & 0,007 & 5,0 & 4,7 & 0,5 & 0,03 & 0,20 & 2,08 & 0,83 & 1,56 \\
\hline Platypodium elegans & 1 & 0,007 & 7,0 & 4,7 & 0,5 & 0,03 & 0,20 & 2,08 & 0,83 & 1,55 \\
\hline Chloroleucon tortum & 1 & 0,007 & 8,0 & 4,7 & 0,5 & 0,03 & 0,18 & 2,08 & 0,83 & 1,54 \\
\hline sp. 1 & 1 & 0,006 & 7,0 & 4,7 & 0,5 & 0,03 & 0,17 & 2,08 & 0,83 & 1,53 \\
\hline sp.3 & 1 & 0,006 & 4,5 & 4,7 & 0,5 & 0,03 & 0,17 & 2,08 & 0,83 & 1,53 \\
\hline Casearia decandra & 1 & 0,006 & 7,5 & 4,7 & 0,5 & 0,03 & 0,16 & 2,04 & 0,08 & 1,52 \\
\hline Cereus jamacaru & 1 & 0,005 & 2,5 & 4,7 & 0,5 & 0,03 & 0,14 & 2,08 & 0,83 & 1,50 \\
\hline Pterogyne nitens & 1 & 0,005 & 6,0 & 4,7 & 0,5 & 0,02 & 0,14 & 2,08 & 0,83 & 1,50 \\
\hline Platymiscium blanchetii & 1 & 0,004 & 4,0 & 4,7 & 0,5 & 0,02 & 0,12 & 2,08 & 0,83 & 1,48 \\
\hline Maclura tinctoria & 1 & 0,004 & 3,0 & 4,7 & 0,5 & 0,02 & 0,11 & 2,08 & 0,83 & 1,46 \\
\hline Lonchocarpus campestris & 1 & 0,004 & 5,0 & 4,7 & 0,5 & 0,02 & 0,10 & 2,08 & 0,83 & 1,45 \\
\hline Tabebuia sp. & 1 & 0,003 & 4,0 & 4,7 & 0,5 & 0,02 & 0,09 & 2,08 & 0,83 & 1,45 \\
\hline Coccoloba schwackeana & 1 & 0,002 & 4,0 & 4,7 & 0,5 & 0,01 & 0,06 & 2,08 & 0,83 & 1,42 \\
\hline Allophyus edulis & 1 & 0,002 & 2,5 & 4,7 & 0,5 & 0,01 & 0,06 & 2,08 & 0,83 & 1,41 \\
\hline Bauhinia cheilantha & 1 & 0,002 & 4,0 & 4,7 & 0,5 & 0,01 & 0,06 & 2,08 & 0,83 & 1,41 \\
\hline Fabaceae sp. & 1 & 0,002 & 5,0 & 4,7 & 0,5 & 0,01 & 0,05 & 2,08 & 0,83 & 1,41 \\
\hline Senna macranthera & 1 & 0,002 & 3,5 & 4,7 & 0,5 & 0,01 & 0,05 & 2,08 & 0,83 & 1,41 \\
\hline
\end{tabular}

Revista Árvore, Viçosa-MG, v.35, n.1, p.131-142, 2011 
Table 3 - List of species sampled in Lapa Pintada dry forest fragment, Capitão Enéas, MG, and its phytosociological parameters, in decreasing sequence of importance value (IV). $\mathrm{N}=$ number of sampled individuals; $\mathrm{BA}=$ basal area; $\mathrm{H}_{\max }=$ maximum height; $\mathrm{AD}=$ absolute density; $\mathrm{RD}=$ relative density; $\mathrm{ADo}=$ absolute dominance; $\mathrm{RDo}=$ relative dominance; $\mathrm{AFr}=$ absolute frequency; RFr= relative frequency.

Tabela 3 - Lista de espécies amostradas na Lapa Pintada, Capitão Enéas, MG, e seus parâmetros fitossociológicos, em ordem decrescente de valor de importância (IV). $N=$ número de indivíduos amostrados; $B A=$ área basal; $H_{\max }=$ altura máxima; $A D=$ densidade absoluta; $R D=$ densidade relativa; $A D o=$ dominância absoluta; $R D o=$ dominância relativa; $A F r=$ frequência absoluta; $R F r=$ frequência relativa .

\begin{tabular}{|c|c|c|c|c|c|c|c|c|c|c|}
\hline Species & $\mathrm{N}$ & $\mathrm{BA}\left(\mathrm{m}^{2}\right)$ & $\mathrm{H}_{\max }(\mathrm{m})$ & $\mathrm{AD}$ & $\mathrm{RD}$ & ADo & RDo & $\mathrm{AFr}$ & $\mathrm{RFr}$ & IV \\
\hline Myracrodruon urundeuva & 21 & 0,419 & 13,00 & 327,3 & 21,9 & 6,53 & 37,70 & 50,00 & 18,20 & 77,80 \\
\hline Combretum duarteanum & 19 & 0,114 & 13,00 & 296,1 & 19,8 & 1,78 & 10,30 & 50,00 & 18,20 & 48,30 \\
\hline Combretum leprosum & 18 & 0,093 & 11,00 & 280,5 & 18,8 & 1,45 & 8,35 & 37,50 & 13,60 & 40,70 \\
\hline Anadenanthera colubrina & 10 & 0,165 & 14,00 & 155,8 & 10,4 & 2,58 & 14,90 & 33,30 & 12,10 & 37,40 \\
\hline Lonchocarpus campestris & 2 & 0,111 & 13,00 & 31,2 & 2,1 & 1,73 & 10,00 & 8,33 & 3,03 & 15,10 \\
\hline Tabebuia roseoalba & 4 & 0,009 & 7,00 & 62,3 & 4,2 & 0,14 & 0,82 & 16,70 & 6,06 & 11,10 \\
\hline Deguellia costata & 2 & 0,051 & 10,00 & 31,2 & 2,1 & 0,80 & 4,63 & 4,17 & 1,52 & 8,23 \\
\hline Schinopsis brasiliensis & 2 & 0,032 & 11,00 & 31,2 & 2,1 & 0,49 & 2,84 & 8,33 & 3,03 & 7,95 \\
\hline Bougainvillea praecox & 1 & 0,051 & 12,00 & 15,6 & 1,0 & 0,79 & 4,58 & 4,17 & 1,52 & 7,14 \\
\hline Piptadenia gonoacantha & 3 & 0,008 & 8,00 & 46,8 & 3,1 & 0,12 & 0,71 & 8,33 & 3,03 & 6,86 \\
\hline Machaerium acutifolium & 2 & 0,008 & 8,00 & 31,2 & 2,1 & 0,12 & 0,71 & 8,33 & 3,03 & 5,82 \\
\hline Sapium obovatum & 2 & 0,004 & 6,00 & 31,2 & 2,1 & 0,06 & 0,32 & 4,17 & 1,52 & 3,92 \\
\hline Pterogyne nitens & 1 & 0,011 & 11,00 & 15,6 & 1,0 & 0,17 & 0,95 & 4,17 & 1,52 & 3,51 \\
\hline Acacia polyphylla & 1 & 0,008 & 8,00 & 15,6 & 1,0 & 0,12 & 0,69 & 4,17 & 1,52 & 3,25 \\
\hline Commiphora leptophloeos & 1 & 0,007 & 8,00 & 15,6 & 1,0 & 0,11 & 0,64 & 4,17 & 1,52 & 3,20 \\
\hline Terminalia sp. & 1 & 0,004 & 10,00 & 15,6 & 1,0 & 0,07 & 0,37 & 4,17 & 1,52 & 2,93 \\
\hline Acacia sp. & 1 & 0,004 & 65,00 & 15,6 & 1,0 & 0,06 & 0,36 & 4,17 & 1,52 & 2,92 \\
\hline Bauhinia rufa & 1 & 0,004 & 4,00 & 15,6 & 1,0 & 0,06 & 0,36 & 4,17 & 1,52 & 2,91 \\
\hline Leucochloron incuriale & 1 & 0,002 & 6,00 & 15,6 & 1,0 & 0,04 & 0,21 & 4,17 & 1,52 & 2,76 \\
\hline Aspidosperma pyrifolium & 1 & 0,002 & 4,00 & 15,6 & 1,0 & 0,04 & 0,21 & 4,17 & 1,52 & 2,76 \\
\hline Bauhinia forficata & 1 & 0,002 & 6,00 & 15,6 & 1,0 & 0,03 & 0,16 & 4,17 & 1,52 & 2,72 \\
\hline Cavanillesia arborea & 1 & 0,002 & 5,00 & 15,6 & 1,0 & 0,03 & 0,16 & 4,17 & 1,52 & 2,72 \\
\hline
\end{tabular}

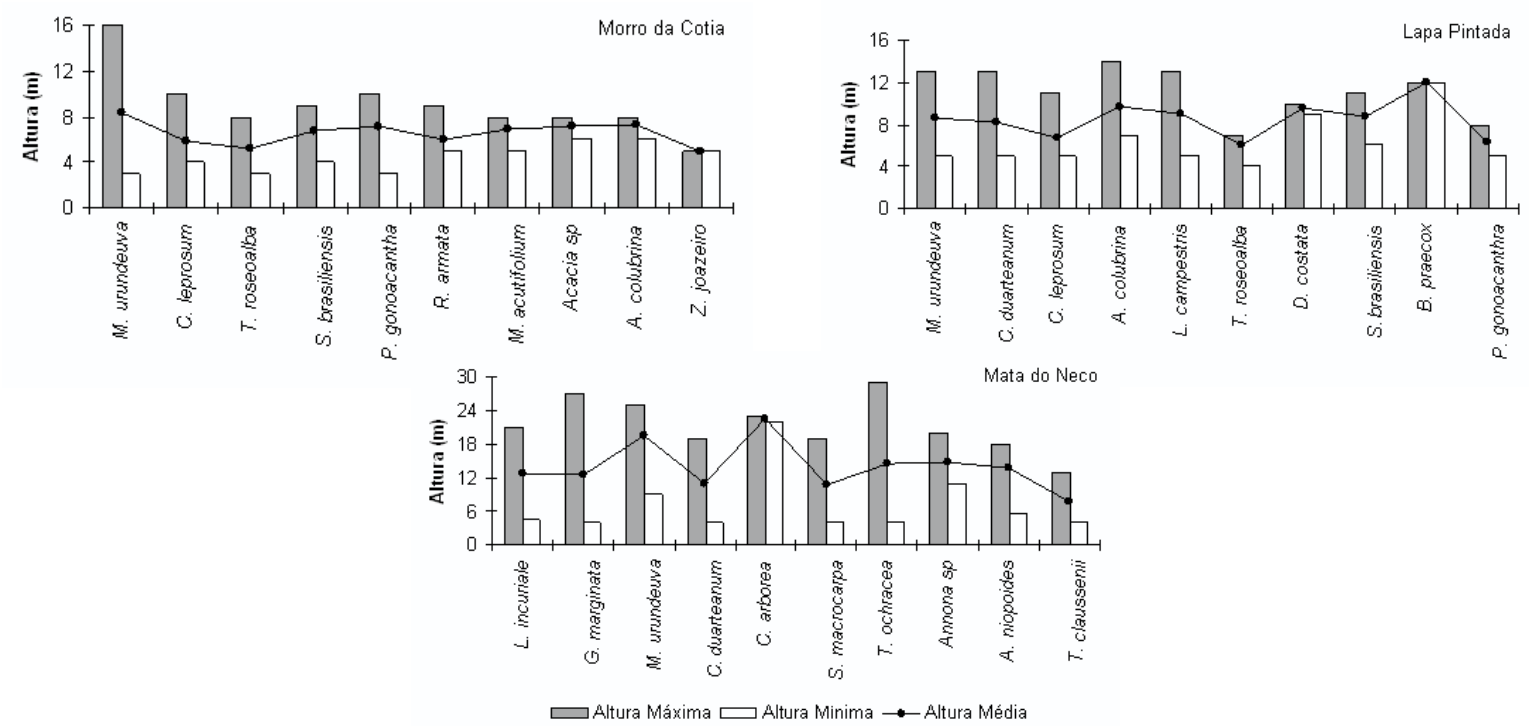

Figure 1 - Graphical representation of the vertical stratification of the ten species registered with the highest IV indexes in the three dry forest fragments sampled in Capitão Enéas, MG.

Figura 1 - Representação gráfica da estratificação vertical das dez espécies registradas com maior índice de VI nos três fragmentos de floresta tropical seca amostrados em Capitão Enéas, MG. 
urundeuva (7.1\%), C. duarteanum (9.4\%) and Cavanillesia arborea (0.9\%). Although only two individuals of $C$. arborea have been sampled, they presented high IV due to its great diameter with average of $108.1 \mathrm{~cm}$. This fragment presents a canopy formed by $M$. urundeuva $(19.4 \mathrm{~m})$ with emergent individuals of $C$. arborea $(22.5 \mathrm{~m})$ and intermediate stratum formed by L. incuriale (12.7 $\mathrm{m}$ ) and G. marginata $(12.6 \mathrm{~m})$. The average of the vertical structure is higher than the other sampled areas (12.4 $\mathrm{m}$ ) and the total density of individuals (578.7 ind.ha ${ }^{-1}$ ) is lower. The diversity $\left(\mathrm{H}^{\prime}=2.78\right)$ and evenness $\left(\mathrm{J}^{\prime}=0.78\right)$ of $\mathrm{MN}$ were higher than the other sampled areas.

In general, the studied areas presented low floristic similarity, being the major figure among MC and LP (0.33) and the minor for MC and MN (0.18). The similarity between the LP and the $\mathrm{MN}$ was also considered low (0.24).

Table 4 - List of species sampled in Mata do Neco dry forest fragment, Capitão Enéas, MG, and its phytosociological parameters, in decreasing sequence of importance value (IV). $\mathrm{N}=$ number of sampled individuals; $\mathrm{BA}=$ basal area; $\mathrm{H}_{\max }=$ maximum height; $\mathrm{AD}=$ absolute density; $\mathrm{RD}=$ relative density; $\mathrm{ADo}=$ absolute dominance; $\mathrm{RDo}=$ relative dominance; $\mathrm{AFr}=$ absolute frequency; $\mathrm{RFr}=$ relative frequency.

Tabela 4 - Lista de espécies amostradas na Mata do Neco, Capitão Enéas, MG, e seus parâmetros fitossociológicos, em ordem decrescente de valor de importância (IV). $N=$ número de indivíduos amostrados; $B A=$ área basal; $H_{\text {max }}=$ altura máxima; $A D=$ densidade absoluta; $R D=$ densidade relativa; $A D o=$ dominância absoluta; $R D o=$ dominância relativa; $A F r=$ frequência absoluta; $R F r=$ frequência relativa.

\begin{tabular}{|c|c|c|c|c|c|c|c|c|c|c|}
\hline Species & $\mathrm{N}$ & $\mathrm{BA}\left(\mathrm{m}^{2}\right)$ & $\mathrm{H}_{\max }(\mathrm{m})$ & $\mathrm{AD}$ & $\mathrm{RD}$ & ADo & RDo & AFr & $\mathrm{RFr}$ & IV \\
\hline$\overline{\text { Leucochloron incuriale }}$ & 53 & 2,089 & 21,00 & 136,9 & 23,7 & 5,40 & 19,00 & 53,60 & 16,90 & 59,50 \\
\hline Goniorrhachis marginata & 29 & 1,701 & 27,00 & 74,9 & 13,0 & 4,39 & 15,50 & 41,10 & 12,90 & 41,40 \\
\hline Myracrodruon urundeuva & 16 & 2,404 & 25,00 & 41,3 & 7,1 & 6,21 & 21,90 & 25,00 & 7,87 & 36,90 \\
\hline Combretum duarteanum & 21 & 0,453 & 19,00 & 54,2 & 9,4 & 1,17 & 4,12 & 26,80 & 8,43 & 21,90 \\
\hline Cavanillesia arborea & 2 & 1,837 & 23,00 & 5,2 & 0,9 & 4,74 & 16,70 & 3,57 & 1,12 & 18,70 \\
\hline Spondias macrocarpa & 13 & 0,376 & 19,00 & 33,6 & 5,8 & 0,97 & 3,42 & 19,60 & 6,18 & 15,40 \\
\hline Tabebuia ochracea & 11 & 0,346 & 29,00 & 28,4 & 5,8 & 0,90 & 3,42 & 17,90 & 6,18 & 15,40 \\
\hline Annona sp. & 8 & 0,274 & 20,00 & 20,7 & 3,6 & 0,71 & 2,49 & 14,30 & 4,49 & 10,60 \\
\hline Albizia niopoides & 9 & 0,280 & 18,00 & 23,2 & 4,0 & 0,72 & 2,55 & 12,50 & 3,93 & 10,50 \\
\hline Trichilia claussenii & 9 & 0,071 & 13,00 & 23,2 & 4,0 & 0,18 & 0,64 & 14,30 & 4,49 & 9,16 \\
\hline Acosmium fallax & 7 & 0,223 & 17,00 & 18,1 & 3,1 & 0,58 & 2,03 & 12,50 & 3,93 & 9,08 \\
\hline Talisia esculenta & 6 & 0,040 & 9,00 & 15,5 & 2,7 & 0,10 & 0,36 & 10,70 & 3,37 & 6,41 \\
\hline Aralia warmingiana & 5 & 0,189 & 19,00 & 12,9 & 2,2 & 0,49 & 1,72 & 5,36 & 1,69 & 5,64 \\
\hline Machaerium acutifolium & 5 & 0,049 & 14,00 & 12,9 & 2,2 & 0,13 & 0,45 & 8,93 & 2,81 & 5,49 \\
\hline Acacia sp. & 4 & 0,044 & 12,00 & 10,3 & 1,8 & 0,11 & 0,40 & 5,36 & 1,69 & 3,87 \\
\hline Syagrus oleraceae & 3 & 0,066 & 20,00 & 7,7 & 1,3 & 0,17 & 0,60 & 5,36 & 1,69 & 3,62 \\
\hline Schinopsis brasiliensis & 2 & 0,090 & 17,00 & 5,2 & 0,9 & 0,23 & 0,82 & 3,57 & 1,12 & 2,84 \\
\hline Machaerium scleroxylon & 2 & 1,205 & 15,00 & 5,2 & 0,9 & 0,21 & 0,73 & 3,57 & 1,12 & 2,75 \\
\hline Cnidoscolus pubescens & 1 & 0,149 & 17,00 & 2,6 & 0,5 & 0,39 & 1,36 & 1,79 & 0,56 & 2,37 \\
\hline Tabebuia roseoalba & 2 & 0,023 & 12,00 & 5,2 & 0,9 & 0,06 & 0,21 & 3,57 & 1,12 & 2,22 \\
\hline Platypodium elegans & 2 & 0,017 & 11,00 & 5,2 & 0,9 & 0,05 & 0,16 & 3,57 & 1,12 & 2,17 \\
\hline sp.2 & 1 & 0,065 & 15,00 & 2,6 & 0,5 & 0,17 & 0,59 & 1,79 & 0,56 & 1,60 \\
\hline Deguelia nitidula & 1 & 0,040 & 11,00 & 2,6 & 0,5 & 0,10 & 0,36 & 1,79 & 0,56 & 1,37 \\
\hline Commiphora leptophloeos & 1 & 0,019 & 7,00 & 2,6 & 0,5 & 0,05 & 0,18 & 1,79 & 0,56 & 1,18 \\
\hline Sapium obovatum & 1 & 0,009 & 5,00 & 2,6 & 0,5 & 0,02 & 0,08 & 1,79 & 0,56 & 1,09 \\
\hline sp.4 & 1 & 0,007 & 11,00 & 2,6 & 0,5 & 0,02 & 0,07 & 1,79 & 0,56 & 1,07 \\
\hline Bauhinia forficata & 1 & 0,007 & 7,00 & 2,6 & 0,5 & 0,02 & 0,07 & 1,79 & 0,56 & 1,07 \\
\hline Erythrina velutina & 1 & 0,006 & 4,50 & 2,6 & 0,5 & 0,02 & 0,06 & 1,79 & 0,56 & 1,07 \\
\hline Sweetia fruticosa & 1 & 0,006 & 10,00 & 2,6 & 0,5 & 0,02 & 0,06 & 1,79 & 0,56 & 1,07 \\
\hline Bougainvillea praecox & 1 & 0,005 & 6,00 & 2,6 & 0,5 & 0,01 & 0,04 & 1,79 & 0,56 & 1,05 \\
\hline Randia armata & 1 & 0,005 & 5,00 & 2,6 & 0,5 & 0,01 & 0,04 & 1,79 & 0,56 & 1,05 \\
\hline Tabernaemontana solanifolia & 1 & 0,005 & 8,00 & 2,6 & 0,5 & 0,01 & 0,04 & 1,79 & 0,56 & 1,05 \\
\hline Campomanesia sp. & 1 & 0,004 & 8,00 & 2,6 & 0,5 & 0,01 & 0,04 & 1,79 & 0,56 & 1,04 \\
\hline Casearia decandra & 1 & 0,002 & 8,00 & 2,6 & 0,5 & 0,01 & 0,02 & 1,79 & 0,56 & 1,02 \\
\hline
\end{tabular}

Revista Árvore, Viçosa-MG, v.35, n.1, p.131-142, 2011 


\section{DISCUSSION}

The richness of species found in this work is similar to the observed by Gentry (1995) that has obtained an average of 64.9 species in 23 areas of Tropical Dry Forest (TDF). In a similar way, Nascimento et al. (2004), Oliveira-Filho et al. (1998), and Salis et al. (2004) found a richness of 52, 60 and 79 species respectively with similar inclusion criteria. However, the richness observed in the areas sampled in this study was lower than the values found by Werneck et al. (2000) of 114 species in a study of a TDF in the Triângulo Mineiro region.

The dominance of Fabaceae is common in TDF (IVANAUSKAS and RODRIQUES, 2000; NASCIMENTO et al., 2004; SALIS et al., 2004; SANTOS et al., 2007; FELFILI et al., 2007; MADEIRA et al., 2009), Cerrado and Caatinga areas (QUEIROZ, 2005). Then the great number of Fabaceae family individuals observed in the sampled fragments can be justified by the localization of studied area in a region of transition of these domains.

The average values of the vertical structure from the "Morro da Cotia" (MC) and "Lapa Pintada" (LP) are in accordance with the results obtained by Silva and Scariot (2004) in dry forests on limestone outcrop and with the average stipulated by Scolforo and Carvalho (2006) of $6.6 \mathrm{~m}$ for TDF areas. However, "Mata do Neco" (MN) presents a high average of vertical structure and high values of basal area, what differentiates it from the other areas. Its flat terrain associated to the inexistence of limestone outcrops promotes the existence of soils with better conditions for water storage like Latosols. Moreover, the growth of the roots is not physically limited by the rocks, what would facilitate the vegetation development. These aspects indicate the importance of edaphic factors in the determination of a TDF structure.

The ten species of greatest IV in MC fit into the ecological group of the pioneers, while MN has mostly secondary species (Table 1 ). These results indicate that $\mathrm{MC}$ is an example of an earlier sucessional stage when compared to MN which represents a later stage. Exclusively in this area, typically pioneering species were found as Celtis iguanaea, which generally occurs in canopy gaps, and Guazuma ulmifolia, that has a high growth tax and is luminosity demanding (OLIVEIRAFILHO et al., 1998). According to Madeira et al. (2009), while a substitution of pioneering species for intermediate and late species occurs, there is an increase in height in the tree community and a closing of the forest canopy.
The abundance of $M$. urundeuva is directly related to the degree of disturbance of the dry forest. This species occurs with higher density in areas where the anthropic activities are recent and with high intensity of disturbance. This fact was also observed by Silva and Scariot (2004) in a dry forest on limestone outcrop in the basin of the Paranã river, in the Goiás State. The pioneering characteristic of this species was also described by Oliveira-Filho et al. (1998) that point out its aspects of light dependence for establishment and growth.

The low floristic similarity among different TDF formations has been pointed out as a natural characteristic even when close fragments are compared (SCARIOT and SEVILHA, 2005). This aspect can still be enhanced by topography and soil conditions, which are important factors that affect the distribution of the species in dry forest (OLIVEIRA-FILHO et al., 1998; SCARIOT and SEVILHA, 2005). TDF patches located on flat areas and deep soils have species that establish themselves in a substrate that has the lowest microclimate variation, while those ones that occur on limestone outcrops present adaptations to the evident irregularity of the terrain where the microclimatic gradient is wider (BRINA, 1998; OLIVEIRA-FILHO et al., 1998). Then the substrate seems to be responsible for a great number of the floristic and structural differences between the sampled fragments, since the precipitation is similar among the studied areas.

The similarity observed between MC and LP may be due to the recent disturbances that both suffered. Possibly this similarity is related to the intensity of the management practices adopted before the abandonment of the area, affecting strongly the natural regeneration process (MADEIRA et al., 2009). In a similar way, the greatest total density and the lowest values of basal area registered in MC and LP are probably due to the similar regeneration time of these areas that would promote the increase in the density of colonizing and of lower height species (WERNECK et al., 2000). Alternatively, the lower height of the individuals in these areas can be explained by the naturally difference existing between dry forests of flat areas and those ones that occur on limestone outcrops (SCARIOT and SEVILHA, 2005). According to Salis et al. (2004), this difference is related to the landscape and to the soil characteristics, which affect differently the draining, the water penetration and its storage in the soil. These

Revista Árvore, Viçosa-MG, v.35, n.1, p.131-142, 2011 
environmental factors of limestone outcrops determinate less favorable conditions to the vegetation growth (PEDRALLI, 1997).

The floristic diversity registered in the studied TDF patches demonstrates the importance of adopting efficient management practices for recovery and preservation of these areas. Each fragment supports a well differentiated local flora due to its particularities of landscape, soil and management history. In this way, the loss of a single fragment would cause negative impacts on the regional flora and, consequently, the loss of associated biodiversity. Moreover, this study supplies information for a better understanding of the factors that determine patterns of species distribution in TDF areas.

\section{ACKNOWLEDGEMENTS}

We thank the people of Santana da Serra, who welcomed us with great attention, and the Laboratório de Ecologia e Propagação Vegetal of Universidade Estadual de Montes Claros - UNIMONTES for the support. Thanks for the grant provided by Fundação de Amparo à Pesquisa de Minas Gerais - FAPEMIG to Y.R.F. Nunes (CRA - BIP-00232-09) and G. S. Tolentino (PROBIC), and by Coordenação de Aperfeiçoamento de Pessoal de Nível Superior - CAPES to F. V. Costa.

\section{REFERENCES}

ANTUNES, F. Z. Caracterização climática. Informe Agropecuário, v.17, n.181, p.15-19, 1994.

\section{APG II - THE ANGIOSPERM PHYLOGENY GROUP. An update of the angiosperm phylogeny group classification for orders and families of flowering plants: APG II. Botanical Journal of the Linnean Society, v.141, p.399-436, 2003.}

ARROYO-MORA, J. P. et al. Quantifying successional stages of tropical dry forests using Landsat ETM+. Biotropica, v.37, p.497-507, 2005.

BRANDÃO, D. O. et al. Regeneração natural de espécies arbóreas em uma área de pastagem vizinha de um fragmento de Floresta Estacional Decidual (Mata Seca) no Norte de Minas Gerais. Revista Brasileira de Biociências, v.5, supl.1, p.546-548, 2007.

Revista Árvore, Viçosa-MG, v.35, n.1, p.131-142, 2011
BRANDÃO, M.; GAVILANES, M. L. Composição florística das áreas recobertas pela Caatinga na área mineira da SUDENE. Informe Agropecuário, v.17, n.181, p.20-33, 1994.

BRINA, A. E. Aspectos da dinâmica da vegetação associada a afloramentos calcários na APA Carste de Lagoa Santa, MG. 1998. Dissertação (Mestrado em Ecologia, Conservação e Manejo da Vida Silvestre) - Universidade Federal de Minas Gerais, Belo Horizonte, 1998. 88p.

CARVALHO, P. E. R. Espécies arbóreas brasileiras. Brasília: Embrapa Informação Tecnológica/Colombo: Embrapa Florestas, 2003. v.1.1039p.

CARVALHO, P. E. R. Espécies arbóreas brasileiras. Brasília: Embrapa Informação Tecnológica, 2006. v.2. 593p.

COTTAM, G.; CURTIS, J. T. The use of distance measures in phytosociological sampling. Ecology, v.37, n.3, p.451-460, 1956.

FELFILI, J. M. et al. Floristic composition and community structure of a seasonally deciduous forest on limestone outcrops in Central Brazil. Revista Brasileira de Botânica, v.30, n.4, p.611-621, 2007.

GENTRY, A. H. Diversity and floristic composition of neotropical dry forests. In: BULLOCK, S. H.; MOONEY, H. A.; MEDINA, E. (Eds.). Seasonally dry topical forests. New York: Cambridge University Press, 1995. p.146-194.

IVANAUSKAS, N. M.; RODRIGUES, R. R. Florística e fitossociologia de remanescentes de floresta estacional decidual em Piracicaba, São Paulo, Brasil. Revista Brasileira de Botânica, v.23, n.3, p.291-304, 2000.

KALÁCSKA, M.; et al. Species composition, similarity and diversity in three successional stages of a seasonally dry tropical forest.

Forest Ecology and Management, v.200, p.227-247, 2004.

KUHLMANN, E. Aspecto gerais da vegetação do alto São Francisco. Revista Brasileira de Geografia, v.13, n.3, p.465-472, 1951. 
LORENZI, H. Árvores brasileiras: manual de identificação e cultivo de plantas arbóreas nativas do Brasil. Nova Odessa: Plantarum, 1992. v.1. 352p.

LORENZI, H. Árvores brasileiras: manual de identificação e cultivo de plantas arbóreas nativas do Brasil. Nova Odessa: Plantarum, 1998. v.2. 368p.

MADEIRA, B. G. et al. Changes in tree and liana communities along a successional gradient in a tropical dry forest in south-eastern Brazil. Plant Ecology, v.201, p.291-304, 2009

MURPHY, P. G.; LUGO, A. E. Ecology of tropical dry forest. Annual Review of Ecology and Systematics, v.17, n.1, p. 67-88, 1986.

MULLER-DOMBOIS, D.; ELLENBERG, H. Aims and methods of vegetation ecology. New York: John Wiley \& Sons, 1974. 547p.

NASCIMENTO, R. T. N.; FELFILI, J. M.; MEIRELLES, M. A. Florística e estrutura da comunidade arbórea de um remanescente de Floresta Estacional Decidual de encosta, Monte Alegre, GO, Brasil. Acta Botanica Brasilica, v.18, n.3, p.650-669, 2004.

NAIME, U. J. Solos da área mineira do polígono das secas. Informe Agropecuário, v.17, n.181, p.10-15, 1994.

OLIVEIRA-FILHO, A. T. et al. Effects of canopy gaps, topography, and soils on the distribution of woody species in a central brazilian deciduous dry forest. Biotropica, v.30, n.3, p.362-375, 1998.

OLIVEIRA-FILHO, A. T. Catálogo das árvores nativas de Minas Gerais: mapeamento e inventário da flora nativa e dos reflorestamentos de Minas Gerais. Lavras: Universidade Federal de Lavras, 2006. 423p.

PEDRALLI, G. Florestas secas sobre afloramentos de calcário em Minas Gerais: florística e fisionomia. Bios, v.5, n.5, p.81-88, 1997.
PENNINGTON, R. T.; LEWIS, G. P.; RATTER, J. A. An overview of the plant diversity, biogeography and conservation of neotropical savannas and seasonally dry forests. In: PENNINGTON, R. T.; RATTER, J. A.; LEWIS, G. P. (Eds.)

Neotropical savannas and dry forests: Plant diversity, biogeography and conservation. Boca Raton: CRC Press, 2005. p.1-29.

QUEIROZ, L. P. The Brazilian Caatinga: Phytogeographical patterns inferred from distribution data of the Leguminosae. In: PENNINGTON, R. T.; RATTER, J. A.; LEWIS, G. P. (Eds.) Neotropical savannas and dry forests: Plant diversity, biogeography and conservation. Boca Raton: CRC Press, 2005. p.113-150.

SALIS, S. M. et al. Fitossociologia de remanescentes de floresta estacional decidual em Corumbá, Estado do Mato Grosso do Sul, Brasil. Revista Brasileira de Botânica, v.27, n.4, p.671-684, 2004.

SÁNCHEZ-AZOFEIFA, G. A. et al. Research priorities for Neotropical Dry Forests.

Biotropica, v.37, p.477-485, 2005.

SANTOS, R. M. et al. Riqueza e similaridade florística de oito fragmentos florestais no norte de Minas Gerais. Revista Árvore, v.31, n.1, p.135-144, 2007.

SCARIOT, A.; SEVILHA, A. C. Biodiversidade, estrutura e conservação de florestas estacionais deciduais no Cerrado. In: SCARIOT, A.; FELFILI, J. M.; SOUZA-SILVA, J. C. (Orgs.). Cerrado: ecologia, biodiversidade e conservação. Brasília: Ministério do Meio Ambiente, 2005. p.121-139.

SCOLFORO, J. R.; CARVALHO, L. M. T. Mapeamento e inventário da flora nativa e dos reflorestamentos de Minas Gerais. Lavras: Universidade Federal de Lavras, 2006. 288p.

SHEPHERD, G. J. FITOPAC 1: Manual do Usuário. Campinas: UNICAMP, 1995. 93p.

SILVA, L. Á.; SCARIOT, A. Comunidade arbórea de uma floresta estacional decídua sobre afloramento calcário na bacia do rio Paraná. Revista Árvore, v.28, n.1, p.61-67, 2004.

Revista Árvore, Viçosa-MG, v.35, n.1, p.131-142, 2011 
VELOSO, H. P.; RANGEL-FILHO, A. L. R.; LIMA, J. C. A. Classificação da vegetação brasileira adaptada a um sistema universal. Rio de Janeiro: IBGE, Departamento de Recursos Naturais e Estudos Ambientais, 1991. 124p.
WERNECK, M. S.; FRANCESCHINELLI, E. V.; TAMEIRÃO-NETO, E. Mudanças na florística e estrutura de uma floresta decídua durante um período de quatro anos (1994-1998), na região do Triângulo Mineiro, MG. Revista Brasileira de Botânica, v.23, n.4, p.399-411, 2000. 